\title{
APPROXIMATE CALCULATION OF CAPACITANCE OF LINES WITH MULTILAYER MEDIUM
}

\author{
Alenka M. MILOVANOVIĆ ${ }^{*}$ — Miroslav M. BJEKIĆ ${ }^{* *}$
}

\begin{abstract}
In this paper calculations of the capacitance per unit length of one or multilayer dielectric lines are presented. Special attention is given to the calculations of the capacitance per unit length of lines with rectangular cross sections, whose electrodes may be in different or the same layers of a two layer dielectric line. For the purpose of performing the above, several numerical methods are used and simple approximate expressions are proposed.

Keywords: lines with rectangular cross section, multilayer medium, numerical methods, capacitance
\end{abstract}

\section{INTRODUCTION}

The problem of calculation of the capacitance per unit length of lines with multilayer medium has been evidenced in both theory and practice. When designing lines and cables with multilayer medium of different geometry, one should often be well acquainted with most accurate values of the capacitance per unit length. Calculation of the capacitance per unit length of lines with multilayer medium can be performed using various analytical and numerical methods such us the Charge Simulation Method (CSM), Finite Element Method (FEM), Equivalent Electrode Method (EEM) [1-7], etc. All these methods, offering different degrees of precision, give results with satisfying accuracy, but also require extensive mathematical work. This is a serious drawback and difficulty that engineers encounter in practice. They are commonly very restricted in terms of time and conditions for comprehensive numerical calculations. The aim of this paper is to provide a review of the applications of different methods for calculation of the capacitance of multilayer lines with a rectangular cross section and to propose a simple procedure for approximate, but sufficiently exact, calculation of capacitance per unit length. This review will be also of great help to PhD students who can use this analysis in research as well as in practice.

\section{LINES WITH ONE LAYER MEDIUM}

The capacitance per unit length of lines with one layer perfect dielectric medium is proportional to the permittivity and can be expressed in general as

$$
C^{\prime}=g^{\prime} \varepsilon,
$$

where $g^{\prime}$ is the coefficient of proportionality which depends on the shape, dimension and mutual position of the electrodes.

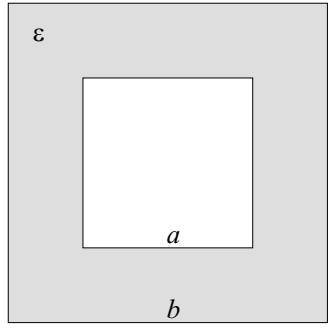

Fig. 1. The square coaxial line

In the case of the square coaxial line, Fig. 1,

$$
g^{\prime}=8 K(k) / K\left(k^{\prime}\right),
$$

where $K(k)$ is the complete elliptic integral of the first kind with modulus $k$,

$$
k=\left(\frac{p-p^{\prime}}{p+p^{\prime}}\right)^{2}=\frac{1-2 p p^{\prime}}{1+2 p p^{\prime}},
$$

and complementary modulus $k^{\prime}, k^{\prime}=\sqrt{1-k^{2}}[7-9] . p$ is the connection with geometry of electrodes,

$$
\frac{K(p)}{K\left(p^{\prime}\right)}=\frac{1-a / b}{1+a / b}, \quad p^{\prime}=\sqrt{1-p^{2}} .
$$

In order to calculate the approximate values of the ratio $K(k) / K\left(k^{\prime}\right)$, Fig. 2, the following simple formula can be applied $[10]$

$$
\frac{K(k)}{K\left(k^{\prime}\right)} \approx \frac{1}{2 \pi} \ln \left(2 \frac{\sqrt{1+k}+\sqrt[4]{4 k}}{\sqrt{1+k}-\sqrt[4]{4 k}}\right) .
$$

Besides expression (2) for the calculation of $g^{\prime}$, the expression given in [11] can also be used

$$
g^{\prime} \approx \begin{cases}8 \frac{0.279+0.721 \frac{a}{b}}{1-a / b}, & 0.25 \leq \frac{a}{b} \leq 0.5, \\ \frac{6.33}{\ln \left(0.956 \frac{b}{a}\right)}, & \frac{a}{b} \leq 0.5 .\end{cases}
$$

\footnotetext{
University of Kragujevac, Technical Faculty of Cacak, Svetog Save 65, Cacak 32000, Serbia, * Department of Electrical Engineering, alenka@tfc.kg.ac.rs; ${ }^{* *}$ Department of Power Engineering
} 


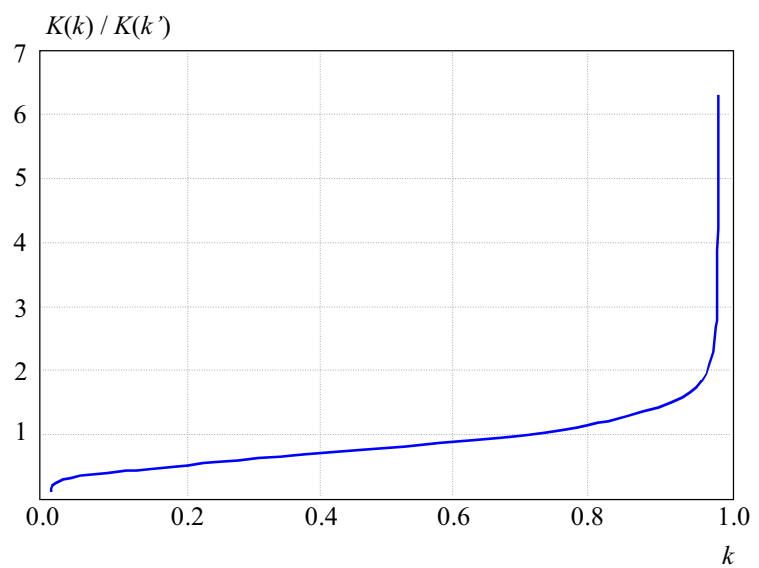

Fig. 2. The ratio $K(k) / K\left(k^{\prime}\right)$

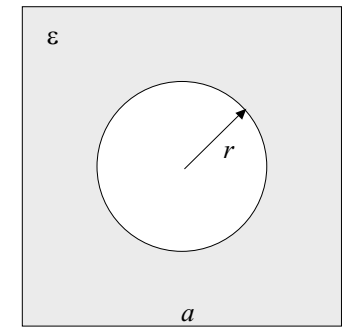

Fig. 3. Coaxial line with circular inner conductor and square outer conductor

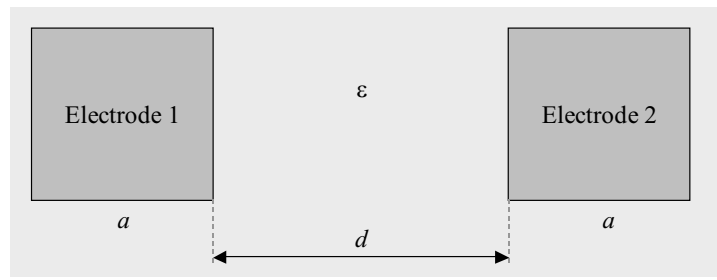

Fig. 4. Two wire line with square cross section

Table 1. The comparison of the results for coefficient of proportionality, in the case of square coaxial line, Fig. 1, for different ratios $a / b$

\begin{tabular}{ccc}
\hline$a / b$ & $g^{\prime} \approx 8 \frac{0.279+0.721 a / b}{1-a / b}$ & $g^{\prime} \approx 8 \frac{K(k)}{K\left(k^{\prime}\right)}$ \\
\hline 0.5 & 10.232000 & 10.236734 \\
0.4 & 7.565333 & 7.561678 \\
0.25 & 4.898666 & 4.844421 \\
0.2 & 4.232000 & 4.134493 \\
\hline$a / b$ & $g^{\prime}(\mathrm{MEE})$ & $g^{\prime}(\mathrm{FEM})$ \\
\hline 0.5 & 10.244873 & 10.243173 \\
0.4 & 7.567525 & 7.568879 \\
0.25 & 4.846910 & 4.846988 \\
0.2 & 4.136304 & 4.136933 \\
\hline
\end{tabular}

Although approximate, expressions (2) and (6) do give satisfactory results, and this can be seen when they are compared with results obtained by other, numerical methods which give results with high precision. For that purpose results were obtained by using Equivalent Electrode Method (Appendix A) and Finite Element Method (Software package Femlab), Table 1.
In the case of the coaxial line with circular inner conductor and square outer conductor, Fig. 3, the coefficient of proportionality is [11]

$$
g^{\prime} \approx \frac{2 \pi}{\ln \left(1.079 \frac{a}{2 r}\right)}
$$

The values of the coefficient of the proportionality, $g^{\prime}$, in the case of a two wire line with a rectangular cross section, Fig. 4, for different ratios of $d / a$ are presented in Table 2 and Fig. 5.

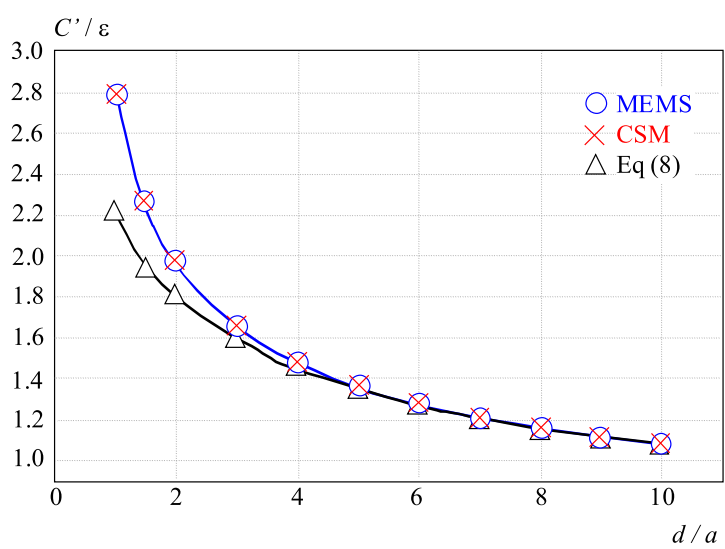

Fig. 5. The capacitance per unit length of the line from Fig. 4, for different ratio $d / a$

The presented results are obtained using the Charge Simulation Method [12], Finite Element Method (Software package Femlab), Modified Equivalent Source Method (MESM) [5] and approximate analytical expression (8) which can be used with satisfying accuracy when $d \gg a[11]$,

$$
g^{\prime} \approx \frac{\pi}{\ln \left[\frac{\pi(d+a)}{2 a}+1\right]} \approx \frac{\pi}{\ln \left[\frac{\pi d}{2 a}+1\right]}, d \gg a
$$

After the capacitance is determined, admittance per unit length of the line having one layer perfect dielectric medium is

$$
\underline{Y}^{\prime}=j \omega C^{\prime}=j \omega \varepsilon g^{\prime}
$$

In the imperfect, linear medium with permittivity $\varepsilon$ and conductivity $\sigma$ not only conductive currents, but also displacement currents can flow [16], so the first Maxwell's equation can be expressed as

$$
\operatorname{rot} \underline{\vec{H}}=(\sigma+j \omega \varepsilon) \underline{\vec{E}}=\underline{\sigma \vec{E}}=j \omega \underline{\varepsilon \vec{E}} .
$$

where: $\underline{\sigma}$ is complex conductivity, $\underline{\sigma}=\sigma+j \omega \varepsilon$; $\underline{\varepsilon}$ is complex permittivity, $\underline{\varepsilon}=\varepsilon\left(1-j \frac{\omega_{c}}{\omega}\right)$ and $\omega_{c}=\sigma / \varepsilon$ is angular frequency when densities of conductive current and displacement current are equal. $\underline{\vec{E}}$ and $\underline{\vec{H}}$ are the complex vectors of electrical and magnetic field strength. 
Table 2. The comparison of the results for capacitance per unit length in the case of the line presented in Fig. 4

\begin{tabular}{cccccccc}
\hline$d / a$ & $\begin{array}{c}C^{\prime} / \varepsilon \\
{[5]}\end{array}$ & $\begin{array}{c}C^{\prime} / \varepsilon \\
(\mathrm{CSM})\end{array}$ & $\begin{array}{c}C^{\prime} / \varepsilon \\
(\mathrm{FEM})\end{array}$ & $d / a$ & $\begin{array}{c}C^{\prime} / \varepsilon \\
{[5]}\end{array}$ & $\begin{array}{c}C^{\prime} / \varepsilon \\
(\mathrm{CSM})\end{array}$ & $\begin{array}{c}C^{\prime} / \varepsilon \\
(\mathrm{Eq} .8)\end{array}$ \\
\hline 0.1 & 13.06355 & 13.06649 & 13.09010 & 1.5 & 2.26644 & 2.26793 & 1.93198 \\
0.2 & 7.65132 & 7.65556 & 7.66498 & 2 & 1.98063 & 1.98147 & 1.80282 \\
0.3 & 5.75052 & 5.75469 & 5.76177 & 3 & 1.66128 & 1.66176 & 1.58225 \\
0.4 & 4.75598 & 4.75991 & 4.76581 & 4 & 1.48049 & 1.48034 & 1.44045 \\
0.5 & 4.13451 & 4.13819 & 4.14316 & 5 & 1.36089 & 1.36117 & 1.34011 \\
0.6 & 3.70462 & 3.70808 & 3.71333 & 6 & 1.27443 & 1.27469 & 1.26441 \\
0.7 & 3.38845 & 3.39027 & 3.39492 & 7 & 1.20820 & 1.20844 & 1.20452 \\
0.8 & 3.14258 & 3.14436 & 3.14844 & 8 & 1.15539 & 1.15561 & 1.15593 \\
0.9 & 2.94572 & 2.94746 & 2.95114 & 9 & 1.11199 & 1.11220 & 1.11567 \\
1 & 2.78393 & 2.78561 & 2.21016 & 10 & 1.07551 & 1.07570 & 1.08116 \\
\hline
\end{tabular}

If the real value of permittivity in (9) is replaced with the complex value, the admitance per unit length of the line having one layer imperfect dielectric medium is

$$
\underline{Y}^{\prime}=j \omega \underline{\varepsilon} g^{\prime}=\underline{\sigma} g^{\prime}=G^{\prime}+j \omega C^{\prime},
$$

where $G^{\prime}=\sigma C^{\prime} / \varepsilon=\omega_{c} C^{\prime}$ is conductance.

\section{LINES WITH TWO LAYER MEDIUM}

In the case of the line with two layer perfect dielectric medium, Figs. 6a and 6b (the electrodes are in different layers of multilayer dielectric line), the capacitance per unit length can be determined by using approximate expression (12), Appendix B,

$$
\frac{1}{C^{\prime}}=\frac{2}{\left(\varepsilon_{1}+\varepsilon_{2}\right) g_{13}^{\prime}}+\frac{\varepsilon_{1}-\varepsilon_{2}}{\varepsilon_{1}+\varepsilon_{2}}\left(\frac{1}{\varepsilon_{2} g_{23}^{\prime}}-\frac{1}{\varepsilon_{1} g_{12}^{\prime}}\right),
$$

where $g_{12}^{\prime}$ and $g_{23}^{\prime}$ are coefficients of the proportionality of the lines which are formed by the existing electrode and the electrode's shield coinciding with separation surface $S$, and $g_{13}^{\prime}$ is a coefficient of proportionality of the line when $\varepsilon_{1}=\varepsilon_{2}$,

$\left.\frac{1}{C^{\prime}}\right|_{\varepsilon_{1} \rightarrow \infty}=\frac{1}{\varepsilon_{2} g_{23}^{\prime}},\left.\frac{1}{C^{\prime}}\right|_{\varepsilon_{2} \rightarrow \infty}=\frac{1}{\varepsilon_{2} g_{12}^{\prime}}$ and $\left.\frac{1}{C^{\prime}}\right|_{\varepsilon_{1}=\varepsilon_{2}}=\frac{1}{\varepsilon_{1} g_{13}^{\prime}}$.

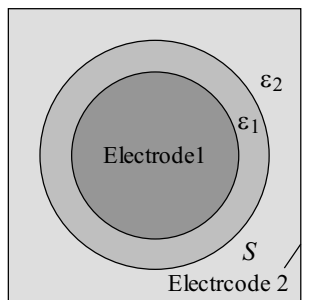

(a)

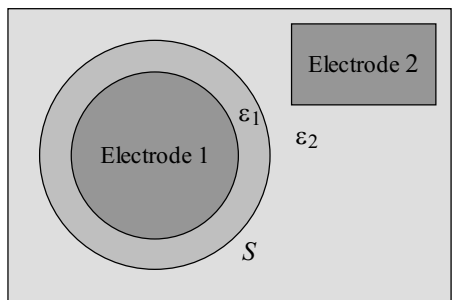

(b)
Fig. 6. Coaxial line with: (a) - circular inner conductor, and (b) square outer conductor
The suggested expression (12) is exact when the surface of separation $S$ is of uniform potential. However, it is useful as a very good approximation in the case when the potential on the surface of separation is not uniform.

The effective permittivity can be calculated as

$$
\varepsilon_{e}=\frac{C^{\prime}}{g_{13}^{\prime}}
$$

and depends on the electric characteristics of the existing layers, conductor's shape and their mutual position. Exceptions are lines having electrodes symmetric in relation to the surface of separation of dielectric layers, where $g_{12}^{\prime}=g_{23}^{\prime}=2 g_{13}^{\prime}$ and

$$
\frac{1}{\varepsilon_{e}}=\frac{1}{2}\left(\frac{1}{\varepsilon_{1}}+\frac{1}{\varepsilon_{2}}\right)
$$

As layers inside the line are imperfect, the admittance per unit length, $\underline{Y}^{\prime}$, is approximately

$$
\frac{1}{\underline{Y^{\prime}}}=\frac{2}{\left(\underline{\sigma}_{1}+\underline{\sigma}_{2}\right) g_{13}^{\prime}}+\frac{\underline{\sigma}_{1}-\underline{\sigma}_{2}}{\underline{\sigma}_{1}+\underline{\sigma}_{2}}\left(\frac{1}{\underline{\sigma}_{2} g_{23}^{\prime}}-\frac{1}{\underline{\sigma}_{1} g_{12}^{\prime}}\right)
$$

where: $Y^{\prime}=G_{e}^{\prime}+j \omega C_{e}^{\prime}, G_{e}^{\prime}=\sigma_{e} g_{13}^{\prime}, C_{e}^{\prime}=\varepsilon_{e} g_{13}^{\prime}, \underline{\sigma}_{1}=$ $\sigma_{1}+j \omega \varepsilon_{1}$ and $\underline{\sigma}_{2}=\sigma_{2}+j \omega \varepsilon_{2} . \sigma_{e}$ and $\varepsilon_{e}$ are effective permittivity and effective conductivity, respectively.

If the lines with two layer imperfect dielectric medium are treated as lines with one layer imperfect dielectric medium, complex effective conductivity is

$$
\underline{\sigma}_{e}=\frac{\underline{Y}^{\prime}}{g_{13}^{\prime}}=\frac{G_{e}^{\prime}+j \omega C_{e}^{\prime}}{g_{13}^{\prime}}=\sigma_{e}+j \omega \varepsilon_{e},
$$

accordingly

$$
\frac{\underline{\sigma}_{1}+\underline{\sigma}_{2}}{2 \underline{\sigma}_{e}}=\alpha+j \beta
$$

where

$$
\begin{gathered}
\varepsilon_{e}=\frac{\alpha\left(\varepsilon_{1}+\varepsilon_{2}\right)+\beta^{\prime}\left(\sigma_{1}+\sigma_{2}\right)}{2\left(\alpha^{2}+\beta^{2}\right)}, \beta^{\prime}=\frac{\beta}{\omega}, \\
\sigma_{e}=\frac{\alpha\left(\sigma_{1}+\sigma_{2}\right)+\omega \beta\left(\varepsilon_{1}+\varepsilon_{2}\right)}{2\left(\alpha^{2}+\beta^{2}\right)},
\end{gathered}
$$




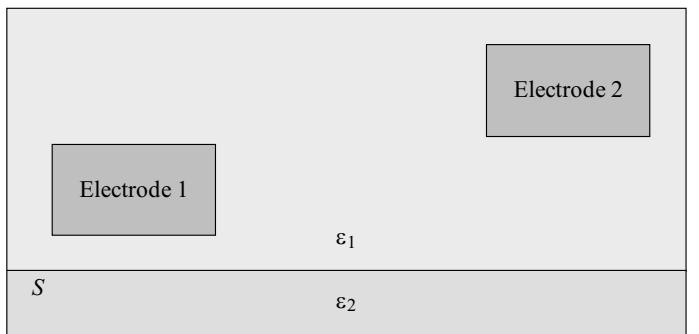

Fig. 7. The line with two layer perfect dielectric medium, where electrodes are in the same layer

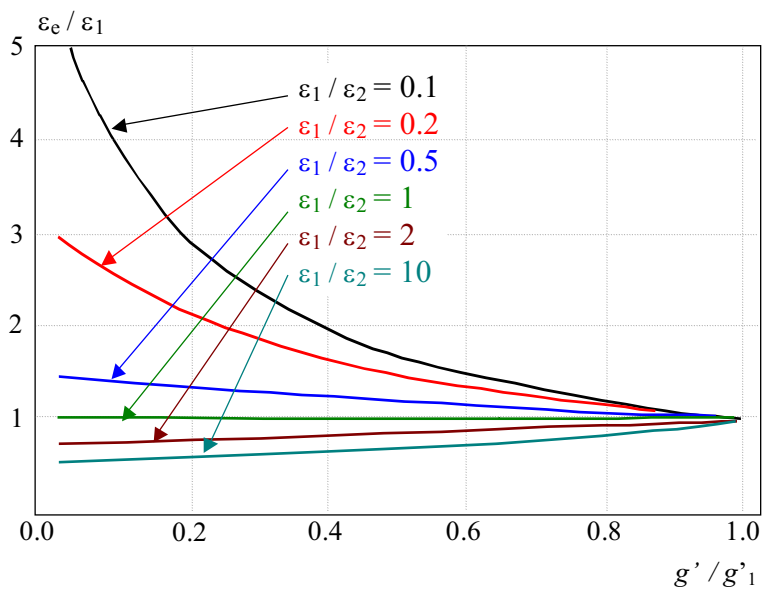

Fig. 8. Effective permittivity of the line where electrodes are in the same layer
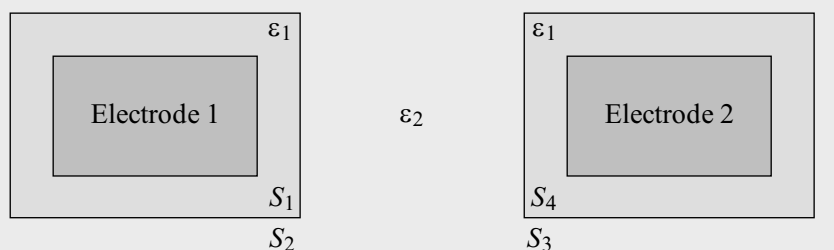

Fig. 9. The line with perfect three layer dielectric medium

$$
\begin{aligned}
\alpha=1- & \frac{\gamma_{1}+\gamma_{2}}{2} \\
& +\frac{\omega_{1} \omega_{2}+\omega^{2}}{2}\left[\frac{\varepsilon_{2}}{\varepsilon_{1}} \frac{\gamma_{1}}{\omega_{2}^{2}+\omega^{2}}+\frac{\varepsilon_{1}}{\varepsilon_{2}} \frac{\gamma_{2}}{\omega_{1}^{2}+\omega^{2}}\right], \\
\beta= & \frac{\omega_{1}-\omega_{2}}{2}\left[\frac{\varepsilon_{1}}{\varepsilon_{2}} \frac{\gamma_{2}}{\omega_{1}^{2}+\omega^{2}}-\frac{\varepsilon_{2}}{\varepsilon_{1}} \frac{\gamma_{1}}{\omega_{2}^{2}+\omega^{2}}\right] \omega, \\
\omega_{1}= & \frac{\sigma_{1}}{\varepsilon_{1}}, \omega_{2}=\frac{\sigma_{2}}{\varepsilon_{2}}, \gamma_{1}=\frac{g_{13}^{\prime}}{g_{12}^{\prime}} \text { and } \gamma_{2}=\frac{g_{13}^{\prime}}{g_{23}^{\prime}}
\end{aligned}
$$

In the case when two layer perfect dielectric medium exists, but the electrodes are in the same layer, Fig. 7, the capacitance per unit length can be determined by using approximate expression (24),

$$
\frac{1}{C^{\prime}}=\frac{2}{\left(\varepsilon_{1}+\varepsilon_{2}\right) g^{\prime}}+\frac{\varepsilon_{2}-\varepsilon_{1}}{\varepsilon_{1}+\varepsilon_{2}} \frac{1}{\varepsilon_{1} g_{1}^{\prime}}
$$

where $g^{\prime}$ and $g_{1}^{\prime}$ are coefficients of proportionality

$$
\left.\frac{1}{C^{\prime}}\right|_{\varepsilon_{2} \rightarrow \infty}=\frac{1}{\varepsilon_{1} g_{1}^{\prime}} \text { and }\left.\frac{1}{C^{\prime}}\right|_{\varepsilon_{1}=\varepsilon_{2}}=\frac{1}{\varepsilon_{1} g^{\prime}} .
$$

When the approximate capacitance is calculated using (24), it is possible to determine the effective permittivity as

$$
\varepsilon_{e}=\frac{C^{\prime}}{g^{\prime}}=\frac{\varepsilon_{1}\left(\varepsilon_{1}+\varepsilon_{2}\right)}{2 \varepsilon_{1}+\left(\varepsilon_{2}-\varepsilon_{1}\right)\left(g^{\prime} / g_{1}^{\prime}\right)} .
$$

As the layers of the lines are imperfect the admittance per unit length is approximately

$$
\begin{gathered}
\frac{1}{\underline{Y}^{\prime}}=\frac{2}{\left(\underline{\sigma}_{1}+\underline{\sigma}_{2}\right) g^{\prime}}+\frac{\underline{\sigma}_{2}-\underline{\sigma}_{1}}{\underline{\sigma}_{1}+\underline{\sigma}_{2}} \frac{1}{\underline{\sigma}_{1} g_{1}^{\prime}}, \\
Y^{\prime}=G_{e}^{\prime}+j \omega C_{e}^{\prime}, \quad G_{e}^{\prime}=\sigma_{e} g^{\prime}, \quad C_{e}^{\prime}=\varepsilon_{e} g^{\prime}, \\
\underline{\sigma}_{1}=\sigma_{1}+j \omega \varepsilon_{1}, \quad \underline{\sigma}_{1}=\sigma_{1}+j \omega \varepsilon_{1} .
\end{gathered}
$$

In this case dielectric complex effective conductivity is:

$$
\underline{\sigma}_{e}=\frac{\underline{Y}^{\prime}}{g^{\prime}}=\frac{\underline{\sigma}_{1}\left(\underline{\sigma}_{1}+\underline{\sigma}_{2}\right)}{2 \underline{\sigma}_{1}+\left(\underline{\sigma}_{2}-\underline{\sigma}_{1}\right)\left(g^{\prime} / g_{1}^{\prime}\right)} .
$$

\section{THE LINE WITH THREE LAYER MEDIUM}

In the case of the line with three layer perfect dielectric medium, such as a two wire line having a rectangular cross section (Fig. 9), the approximate expression for calculation of the capacitance per unit length and effective permittivity are

$$
\frac{1}{C^{\prime}}=\frac{2}{\left(\varepsilon_{1}+\varepsilon_{2}\right) g_{14}^{\prime}}+\frac{\varepsilon_{1}-\varepsilon_{2}}{\varepsilon_{1}+\varepsilon_{2}}\left[\frac{1}{\varepsilon_{2} g_{23}^{\prime}}-\frac{1}{\varepsilon_{1}}\left(\frac{1}{g_{12}^{\prime}}+\frac{1}{g_{34}^{\prime}}\right)\right]
$$

and

$$
\varepsilon_{e}=C^{\prime} / g_{14}^{\prime},
$$

where $g_{14}^{\prime}$ and $g_{23}^{\prime}$ are determined for the line composed by conductors defined by surfaces $S_{1} \leftrightarrow S_{4}$, respectively $S_{2} \leftrightarrow S_{3}$ and $g_{12}^{\prime}, g_{34}^{\prime}$ for the coaxial line defined by surfaces $S_{1} \leftrightarrow S_{2}$, respectively $S_{3} \leftrightarrow S_{4}$.

\section{EXAMPLES}

The application of the proposed approximate expressions (12), (24), and (29) will be illustrated through several examples and the results obtained will be compared with results obtained by using different numerical techniques.

EXAMPLE 1. Results for effective permittivity of square coaxial line with two layer perfect dielectric medium, Fig. 10a, and three layer perfect dielectric medium Fig. 10b, obtained using proposed approximate expressions (12) and (29) are shown in Figs. 11 and 12. Some of the results obtained are compared with results obtained using FEM (Software package Femlab) in Table 3.

Coefficients of proportionality $g_{12}^{\prime}, g_{23}^{\prime}, g_{13}^{\prime}$ in the case of the line from Fig. 10a and $g_{12}^{\prime}, g_{23}^{\prime}, g_{34}^{\prime}, g_{14}^{\prime}$ in the case of the line from Fig. 10b are determined using expression (2). The agreement of the results is very good. 


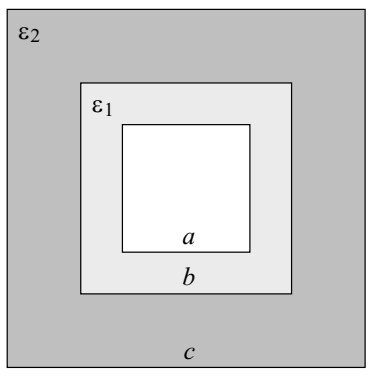

(a)

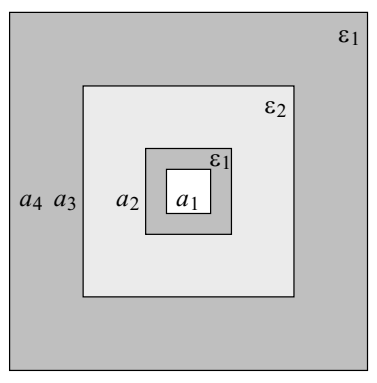

(b)
Fig. 10. Square coaxial line with multilayer dielectric medium

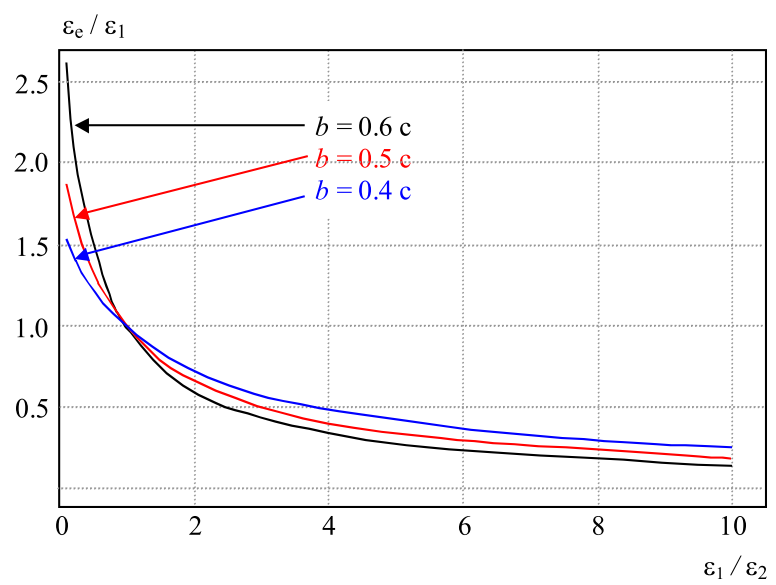

Fig. 11. Effective permittivity of square coaxial line with two layer perfect dielectric medium (Fig. 10a) for different ratios $b / c$ and $c=4 a$

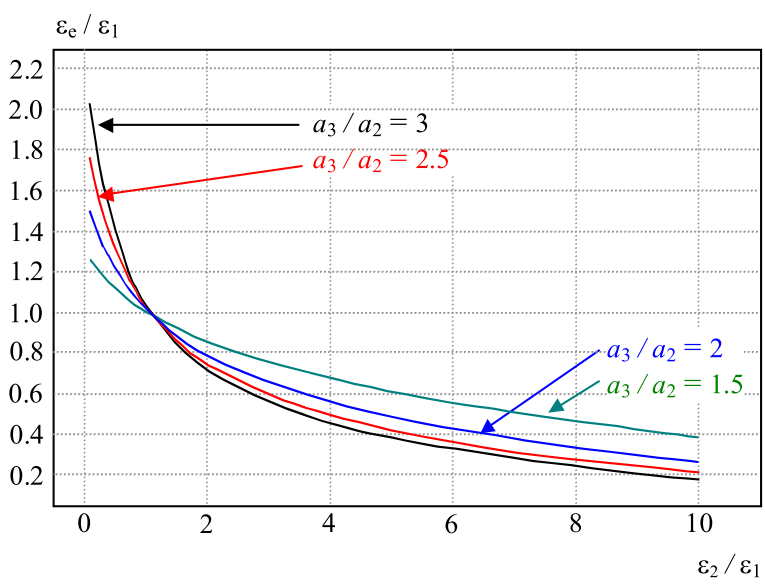

Fig. 12. Effective permittivity of square coaxial line with three layer dielectric medium (Fig. 10b) for different ratios $a_{3} / a_{2}$ and $a_{2} / a_{1}=2, a_{4} / a_{2}=4$

Table 3. The comparison of the results for effective permittivity of the coaxial line, Fig. 10b, when $a_{4} / a_{3}=a_{3} / a_{2}=a_{2} / a_{1}=2$

\begin{tabular}{ccc}
\hline$\varepsilon_{1} / \varepsilon_{2}$ & $\varepsilon_{e} / \varepsilon_{1}(\mathrm{FEM})$ & $\varepsilon_{e} / \varepsilon_{1}(\mathrm{Eq} .29$ and 30$)$ \\
\hline 0.1 & 1.50866 & 1.51188 \\
0.2 & 1.41750 & 1.42134 \\
0.5 & 1.21535 & 1.21813 \\
1 & 1 & 1 \\
2 & 0.75706 & 0.75000 \\
5 & 0.43788 & 0.43788 \\
10 & 0.26045 & 0.26058 \\
\hline
\end{tabular}

EXAMPLE 2. The dependency of effective permittivity of a rectangular coaxial line with two layer perfect dielectric medium against the ratio $\varepsilon_{2} / \varepsilon_{1}$ for the different ratios $a / b, a / b=a_{1} / b_{1}=a_{2} / b_{2}$ and $a_{1} / a=a_{2} / a_{1}=2$ is shown in Fig. 13. The coefficients of proportionality $g_{12}^{\prime}$, $g_{23}^{\prime}$ and $g_{13}^{\prime}$ are determined using EEM.

Also, the coefficients of proportionality can be determined using the analytical expressions given in [11]. For example, for the rectangular coaxial line presented in Fig. 14 expression (31) is suggested

$$
g^{\prime}=\frac{2 \pi}{\ln \frac{1+w / d}{b / d+t / d}}, \quad d>3 t, \quad w>1.25 b .
$$

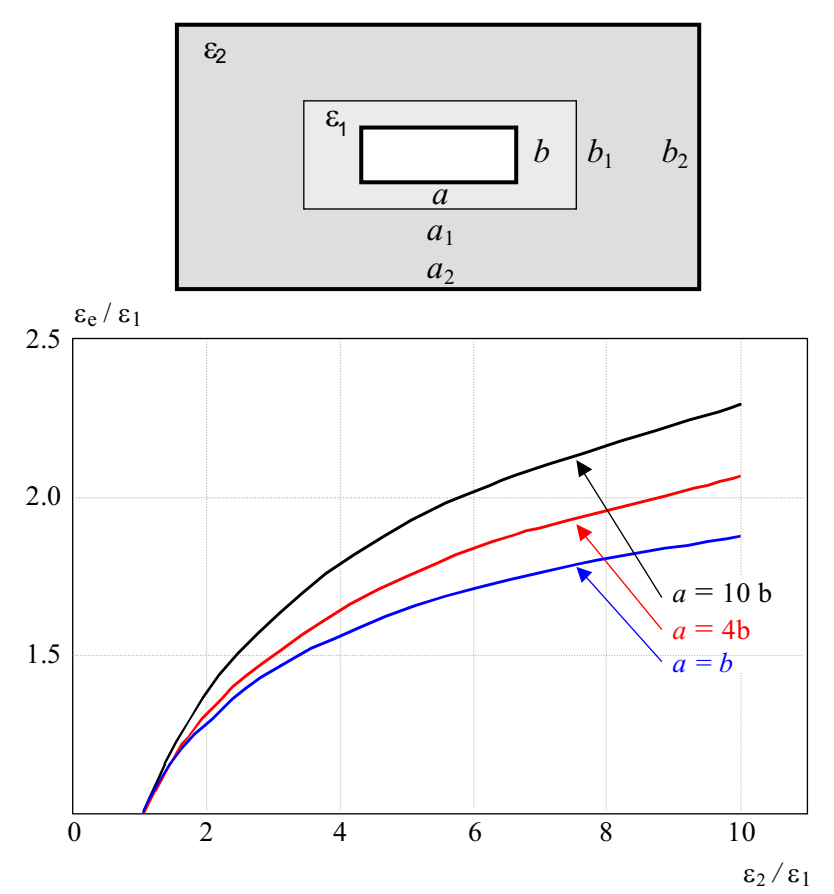

Fig. 13. Effective permittivity of the rectangular coaxial line with two layer perfect dielectric medium

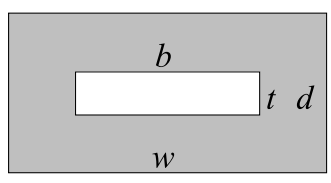

Fig. 14. Rectangular coaxial line

EXAmple 3. For a coaxial line having a circular inner conductor and square outer conductor (Fig. 15) coefficients of proportionality $g_{23}^{\prime}$ and $g_{13}^{\prime}$ are determined using expression (7) and

$$
g_{12}^{\prime}=\frac{2 \pi}{\ln \left(r_{2} / r_{1}\right)}
$$

The results for effective permittivity of the coaxial line obtained using expressions (12) and (14) are compared with results obtained using FEM [Software package FEMM] in Table 4. 
Table 4. Effective permittivity of the coaxial line presented in Fig. 15 , for different ratios $\varepsilon_{1} / \varepsilon_{2}$ and $a / 2 r_{2}$, when $a / 2 r_{1}=4$

\begin{tabular}{ccccccccccc}
\hline & \multicolumn{10}{c}{$\varepsilon_{e} / \varepsilon_{1}$} \\
$\varepsilon_{1} / \varepsilon_{2}$ & \multicolumn{1}{c}{$a / r_{2}=8 / 7$} & \multicolumn{1}{c}{$a / r_{2}=8 / 6$} & \multicolumn{2}{c}{$a / r_{2}=8 / 5$} & $a / r_{2}=8 / 4$ & \multicolumn{2}{c}{$a / r_{2}=8 / 3$} \\
\cline { 2 - 10 } & Eq. (12) & FEM & Eq. (12) & FEM & Eq. $(12)$ & FEM & Eq. $(9)$ & FEM & Eq. $(9)$ & FEM \\
\hline 0.1 & 1.1480 & 1.1474 & 1.2884 & 1.2880 & 1.5062 & 1.5059 & 1.8989 & 1.8970 & 2.8608 & 2.8605 \\
0.2 & 1.1295 & 1.1286 & 1.2484 & 1.2480 & 1.4259 & 1.4257 & 1.7262 & 1.7263 & 2.3706 & 2.3705 \\
0.5 & 1.0772 & 1.0762 & 1.1420 & 1.1416 & 1.2296 & 1.2294 & 1.3568 & 1.3568 & 1.5658 & 1.5658 \\
1 & 1 & 1 & 1 & 1 & 1 & 1 & 1 & 1 & 1 & 1 \\
2 & 0.8747 & 0.8775 & 0.8008 & 0.8015 & 0.7282 & 0.7283 & 0.6553 & 0.6554 & 0.5805 & 0.5805 \\
5 & 0.6356 & 0.6438 & 0.5013 & 0.5027 & 0.40104 & 0.40128 & 0.32216 & 0.32222 & 0.2570 & 0.2570 \\
10 & 0.4367 & 0.4466 & 0.3088 & 0.3101 & 0.2293 & 0.2295 & 0.1744 & 0.1744 & 0.1333 & 0.1333 \\
\hline
\end{tabular}

Table 5. Effective permittivity of coaxial line presented in Fig. 16, for different ratios $\varepsilon_{1} / \varepsilon_{2}$ and $a / a_{1}$, when $a / 2 r=4$
Table 6. Comparison of the results for $C^{\prime} / \varepsilon_{2}$, when $d_{1} / a_{1}=18$ and $d_{2} / a_{2}=8.5$

\begin{tabular}{ccccccccc}
\hline & \multicolumn{8}{c}{$\varepsilon_{e} / \varepsilon_{1}$} \\
\cline { 2 - 8 }$\varepsilon_{1} / \varepsilon_{2}$ & \multicolumn{1}{c}{$a / a_{1}=8 / 6$} & \multicolumn{2}{c}{$a / a_{1}=8 / 5$} & \multicolumn{2}{c}{$a / a_{1}=8 / 4$} & $a / a_{1}=8 / 3$ \\
\cline { 2 - 9 } & Eq. (12) & FEM & Eq. (12) & FEM & Eq. (12) & FEM & Eq. (12) & FEM \\
\hline 0.1 & 1.2096 & 1.2132 & 1.3884 & 1.4026 & 1.7306 & 1.7357 & 2.4927 & 2.5065 \\
0.2 & 1.1782 & 1.1836 & 1.3267 & 1.3394 & 1.5923 & 1.5988 & 2.1218 & 2.1354 \\
0.5 & 1.0997 & 1.1052 & 1.1173 & 1.1843 & 1.2953 & 1.2999 & 1.4826 & 1.4892 \\
1 & 1 & 1 & 1 & 1 & 1 & 1 & 1 & 1 \\
2 & 0.8577 & 0.8402 & 0.7764 & 0.7708 & 0.6955 & 0.6923 & 0.6130 & 0.6104 \\
5 & 0.6125 & 0.5910 & 0.4705 & 0.4647 & 0.3683 & 0.3657 & 0.2869 & 0.2852 \\
10 & 0.4180 & 0.3957 & 0.2853 & 0.2820 & 0.2074 & 0.2062 & 0.1526 & 0.1519 \\
\hline
\end{tabular}

\begin{tabular}{ccc}
\hline$\varepsilon_{1} / \varepsilon_{2}$ & $\begin{array}{c}C^{\prime} / \varepsilon_{2} \\
\text { Eq. }(29)\end{array}$ & $\begin{array}{c}C^{\prime} / \varepsilon_{2} \\
\text { FEM }\end{array}$ \\
\hline 0.1 & 0.34979 & 0.35282 \\
0.2 & 0.53224 & 0.53597 \\
0.5 & 0.77840 & 0.78087 \\
1 & 0.92508 & 0.92482 \\
2 & 1.02574 & 1.02291 \\
5 & 1.10148 & 1.09761 \\
10 & 1.13058 & 1.12723 \\
\hline
\end{tabular}

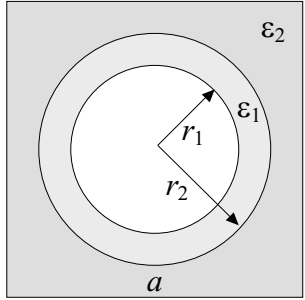

Fig. 15. Coaxial line having a circular inner conductor and square outer conductor

EXAMPLE 4. For a coaxial line presented in Fig. 16, coefficients of proportionality $g_{12}^{\prime}, g_{13}^{\prime}$ and $g_{23}^{\prime}$ are determined using expressions $(7)$ and $(2)$ respectively. The comparison of the results for effective permittivity of the coaxial line, obtained using expressions (12) and (14) and using FEM (Software package FEMM) is presented in Table 5 .

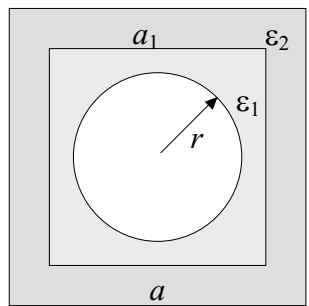

Fig. 16. Coaxial line having a circular inner conductor and square outer conductor

EXAMPLE 5. In the case of the line presented in Fig. 17 the coefficients of proportionality $g_{12}^{\prime}, g_{34}^{\prime}$ and $g_{23}^{\prime}$, $g_{14}^{\prime}$ are determined using expression (2) and expression (8) respectively. Obtained results are presented in Table 6 and Fig. 18 .

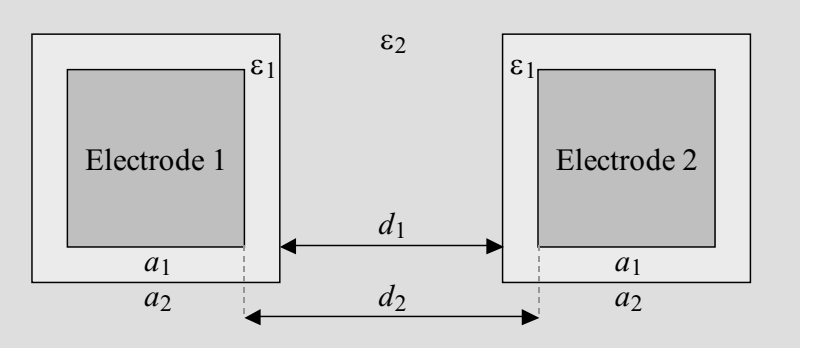

Fig. 17. The line with three layer dielectric medium

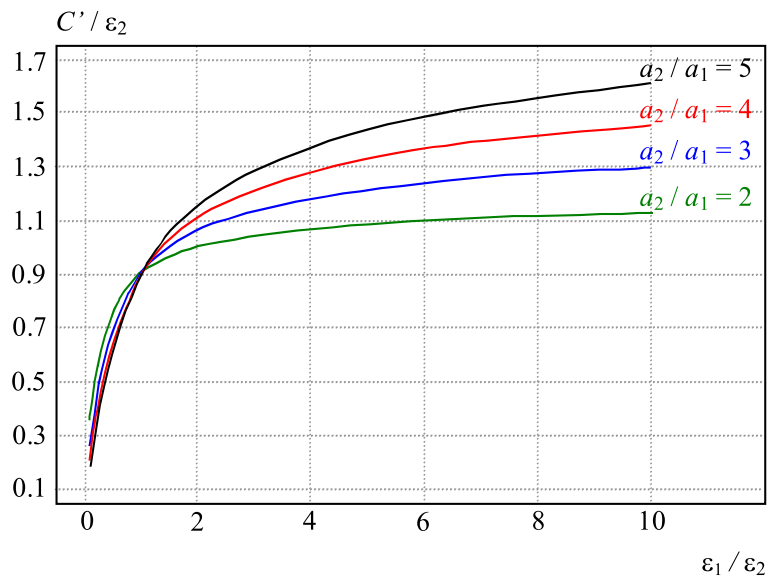

Fig. 18. The ratio $C^{\prime} / \varepsilon_{2}$ for different ratios $a_{1} / a_{2}$ and $\varepsilon_{1} / \varepsilon_{2}$, when $d_{1} / a_{1}=18$ 
Table 7. Comparison of the results for $C^{\prime} / \varepsilon_{0}$, when $d / a=10$, Fig. 19

\begin{tabular}{ccccccc}
\hline & \multicolumn{6}{c}{$C^{\prime} / \varepsilon_{0}$} \\
\cline { 2 - 7 }$\varepsilon_{1} / \varepsilon_{2}$ & \multicolumn{3}{c}{$h / a=2$} & \multicolumn{3}{c}{$h / a=1$} \\
\cline { 2 - 7 } & Eq. (24) & CSM & FEM & Eq. (24) & CSM & FEM \\
\hline 0.1 & 1.432218 & 1.431073 & 1.434849 & 1.742326 & 1.736781 & 1.742185 \\
0.2 & 1.349398 & 1.347887 & 1.353017 & 1.562958 & 1.556347 & 1.563040 \\
0.4 & 1.236992 & 1.235595 & 1.242263 & 1.3453198 & 1.339980 & 1.347690 \\
0.5 & 1.197104 & 1.195929 & 1.203049 & 1.2743402 & 1.270006 & 1.277330 \\
0.8 & 1.113336 & 1.112893 & 1.120840 & 1.1346555 & 1.133158 & 1.141287 \\
1 & 1.075700 & 1.075700 & 1.083968 & 1.075700 & 1.075700 & 1.083874 \\
\hline
\end{tabular}

EXAMPLE 6. The next example presents the line in which the electrodes are in the same layer of the two layer dielectric medium, Fig. 19. The capacitance per unit length is determined using approximate expression (24), CSM and FEM (Software package Femlab). For calculation $g^{\prime}$ and $g_{1}^{\prime}$ CSM is also used. The obtained results are compared in Table 7 .

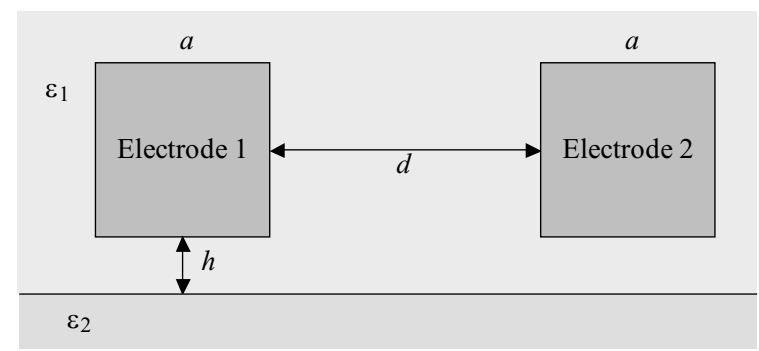

Fig. 19. Two wire line above infinite dielectric surface

\section{CONCLUSION}

This paper presents an instructive review of different techniques for calculations of the capacitance per unit length of lines with multilayer medium, especially lines with rectangular cross section. Several numerical methods (CSM, EEM, MESM) and two program packages $[17,18]$ are used, and some simple equations are proposed which permit an approximate, but sufficiently accurate, evaluation of the capacitances. Expressions for effective permittivity and conductivity are also suggested. The proposed expressions (12), (24) and (29) are postulated and their validity is then tested against several characteristic examples. A very good agreement between obtained results is demonstrated. It is worth mentioning, moreover, that the expressions are always exact in cases of one layer medium, in cases when the surface of separation of the existing layer is of uniform potential as well as in cases when permittivity of one layer has a high value and this medium behaves as a conductive medium. The proposed expressions can simplify the solving of problems as and when they present, and represent a useful tool in everyday engineering practice.

\section{APPENDIX A}

\section{A.1 Equivalent Electrode Method Application}

The basic idea of the method [3,4] is that an arbitrarily shaped electrode can be replaced by a finite system of the equivalent electrodes (EE) located on the body surface. The radius of EE is equal to the equivalent radius of electrode part which it substitutes. Also, the potential and charge of EE and of the real electrode part are equal. So, it is possible using the boundary condition that the electrode is equipotential, and forms a system of linear equations with charge densities of EE as unknowns. After solving this system, unknown charge densities are determined and any other quantity of interest can be easily calculated in a standard way.

In the case when the system has several electrodes, or when a multilayer medium exists, it is convenient to use Green's functions for some electrodes or for a stratified medium. In the case of a square coaxial line Fig. A1 the interior electrode is replaced by $N$ cylindrical EE, with a circular cross section having the radius $a_{e 1}$, charged by line charge per unit length $q_{n}^{\prime}, n=1,2, \ldots, N$. The radius of the $\mathrm{EE}$ is equal to the equivalent radius of the electrode part which it substitutes, in this case thin flat strip conductor, $a_{e 1}=\Delta x_{1} / 4=a / 4 N$.

The position of these EE are $x= \pm x_{n}, y= \pm y_{n}$,

$$
\begin{aligned}
x_{n}=\Delta x_{1} / 2+(n-1) \Delta x_{1}, y_{n}= & a / 2, \\
& n=1,2, \ldots, N / 2
\end{aligned}
$$

and $x_{n}=a / 2, y_{n}=a / 2-\left(\Delta x_{1} / 2+(n-1) \Delta x_{1}\right)$,

$$
n=N / 2, N / 2+1, \ldots, N \text {. }
$$

In a similar way, the shield is replaced by $M$ cylindrical conductors (EE), of circular cross section having the radius $a_{e 2}=\Delta x_{2} / 4=b / 4 M$, charged by $q_{m}^{\prime}$, $m=1,2, \ldots, M$.

The axes of these EE are $x= \pm x_{m}, y= \pm y_{m}$,

$$
\begin{aligned}
x_{m}=\Delta x_{2} / 2+(m-1) \Delta x_{2}, y_{m} & =b / 2, \\
m & =1,2, \ldots, M / 2
\end{aligned}
$$




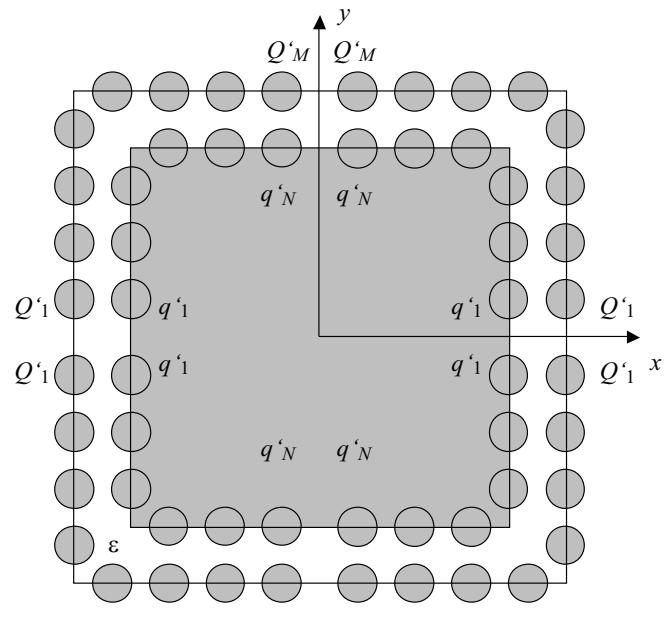

Fig. 20. The square coaxial line

and $x_{m}=b, y_{m}=b / 2-\left(\Delta x_{2} / 2+(m-1) \Delta x_{2}\right)$,

$$
m=M / 2, M / 2+1, \ldots, M \text {. }
$$

So, the total number of the EE accordingly unknown charges having to be determined is $N+M$. Using the condition that equivalent electrodes have the same potential as the electrodes they represent, $\varphi_{1}$ and $\varphi_{2}, \varphi_{1}-\varphi_{2}=U$, system of linear equations are obtained

$$
\begin{array}{r}
U+\varphi_{2}=-\sum_{n=1}^{N} \frac{q_{n}^{\prime}}{2 \pi \varepsilon} \ln \left[r_{1} r_{2} r_{3} \sqrt{r_{4}^{2} a_{e 1}^{2} \delta_{m n}}\right] \\
-\sum_{m=1}^{M} \frac{q_{m}^{\prime}}{2 \pi \varepsilon} \ln \left[r_{1}^{\prime} r_{2}^{\prime} r_{3}^{\prime} \sqrt{r_{4}^{\prime 2} a_{e 2}^{2} \delta_{m n}}\right] \\
x=x_{n}, y=y_{n}, n=1,2, \ldots, N \\
\varphi_{2}=-\sum_{n=1}^{N} \frac{q_{n}^{\prime}}{2 \pi \varepsilon} \ln \left[r_{1} r_{2} r_{3} \sqrt{r_{4}^{2} a_{e 1}^{2} \delta_{m n}}\right] \\
-\sum_{m=1}^{M} \frac{q_{m}^{\prime}}{2 \pi \varepsilon} \ln \left[r_{1}^{\prime} r_{2}^{\prime} r_{3}^{\prime} \sqrt{r_{4}^{\prime 2} a_{e 2}^{2} \delta_{m n}}\right] \\
x=x_{m}, y=y_{m}, m=1,2, \ldots, M . \\
\sum_{n=1}^{N} q_{n}^{\prime}+\sum_{m=1}^{M} q_{m}^{\prime}=0
\end{array}
$$

$r_{1}^{2}=\left(x+x_{n}\right)^{2}+\left(y-y_{n}\right)^{2}, r_{1}^{\prime}=\left(x+x_{m}\right)^{2}+\left(y-y_{m}\right)^{2}$, $r_{2}^{2}=\left(x-x_{n}\right)^{2}+\left(y+y_{n}\right)^{2}, r_{2}^{\prime}=\left(x-x_{m}\right)^{2}+\left(y+y_{m}\right)^{2}$, $r_{3}^{2}=\left(x+x_{n}\right)^{2}+\left(y+y_{n}\right)^{2}, r_{1}^{\prime}=\left(x+x_{m}\right)^{2}+\left(y+y_{m}\right)^{2}$, $r_{4}^{2}=\left(x-x_{n}\right)^{2}+\left(y-y_{n}\right)^{2}, r_{2}^{\prime}=\left(x-x_{m}\right)^{2}+\left(y-y_{m}\right)^{2}$,

and $\delta_{m n}$ is Kronecker symbol.
After solving linear equation (A5-A7) the unknown line charges of the EE are determined and the capacitance per unit length can be calculated as

$$
C^{\prime}=\frac{4 \sum_{n=1}^{N} q_{n}^{\prime}}{U}
$$

\section{APPENDIX B}

\section{B.1 Determination of the proposed expression}

In the case of the line with two layer perfect dielectric medium, Figs. 6a and 6b, when separation surface $S$ is uniform potential, the capacitance per unit length can be exactly determined as

$$
\frac{1}{C^{\prime}}=\frac{1}{C_{12}^{\prime}}+\frac{1}{C_{23}^{\prime}}=\frac{1}{\varepsilon_{1} g_{12}^{\prime}}+\frac{1}{\varepsilon_{2} g_{23}^{\prime}},
$$

where $g_{12}^{\prime}$ and $g_{23}^{\prime}$ are coefficients of the proportionality of the lines which are formed by the existing electrode and the electrode's shield coinciding with surface $S$. After certain elementary transformation, and considering that the

$$
\frac{1}{g_{13}^{\prime}}=\frac{1}{g_{12}^{\prime}}+\frac{1}{g_{23}^{\prime}},
$$

where $\frac{1}{g_{13}^{\prime}}$ is a coefficient of proportionality of the line when $\varepsilon_{1}=\varepsilon_{2}$,

$$
\frac{1}{C^{\prime}}=\frac{\varepsilon_{1}-\varepsilon_{2}}{\varepsilon_{1}+\varepsilon_{2}}\left(\frac{1}{\varepsilon_{1} g_{12}^{\prime}}+\frac{1}{\varepsilon_{2} g_{23}^{\prime}}\right)+\frac{1}{\varepsilon_{1}+\varepsilon_{2}}\left(\frac{1}{g_{13}^{\prime}}-\frac{1}{g_{23}^{\prime}}\right),
$$

the expression (B1) can be expressed in form of (B4) as is proposed in this paper

$$
\frac{1}{C^{\prime}}=\frac{2}{\left(\varepsilon_{1}+\varepsilon_{2}\right) g_{13}^{\prime}}+\frac{\varepsilon_{1}-\varepsilon_{2}}{\varepsilon_{1}+\varepsilon_{2}}\left(\frac{1}{\varepsilon_{2} g_{23}^{\prime}}-\frac{1}{\varepsilon_{1} g_{12}^{\prime}}\right) .
$$

For calculations of the capacitance per unit length, in the case when two layer perfect dielectric medium exists, but the electrodes are in the same layer of multilayer dielectric line, Fig. 7 expression (B5) is proposed

$$
\frac{1}{C^{\prime}}=\frac{2}{\left(\varepsilon_{1}+\varepsilon_{2}\right) g^{\prime}}+\frac{\varepsilon_{2}-\varepsilon_{1}}{\varepsilon_{1}+\varepsilon_{2}} \frac{1}{\varepsilon_{1} g_{1}^{\prime}} .
$$

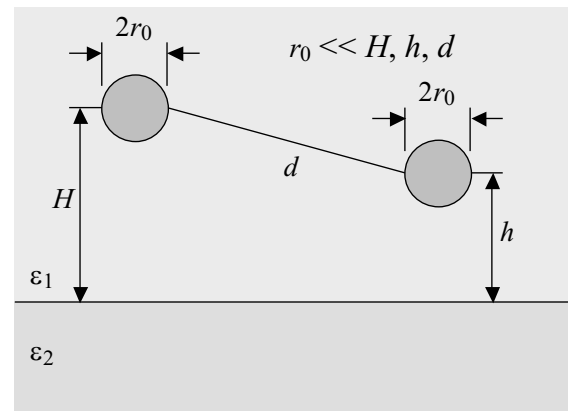

Fig. 21. Two wire line above infinite dielectrical surface 
This expression is formed according to the expression (B4) and firstly, we can check its accuracy in example of a two wire line above infinite dielectrical surface presented in Fig. B1, for which analytical expression exists

$$
\frac{1}{C^{\prime}}=\frac{1}{\varepsilon_{1} \pi}\left(\ln \frac{d}{r_{0}}+\frac{\varepsilon_{2}-\varepsilon_{1}}{\varepsilon_{1}+\varepsilon_{2}} \ln \frac{2 \sqrt{h H}}{\sqrt{d^{2}+4 h H}}\right) .
$$

In this case the coefficients of proportionality $g^{\prime}$ and $g_{1}^{\prime}$ are

$$
g^{\prime}=\frac{\pi}{\ln \left(d / r_{0}\right)} \text { and } g_{1}^{\prime}=\frac{\pi}{\ln \left(2 \sqrt{h H} / r_{0} \sqrt{d^{2}+4 h H}\right)}
$$

Expression (29) proposed for the example of the line with three layer perfect dielectric medium, Fig. 9 is obtained similarly as expressions (B4) and (B5).

\section{Acknowledgement}

The authors of this paper are very grateful to their colleagues from the University of Technology Ilmenau, Germany, who provided access to the software package Femlab during the authors' stay at their University.

\section{REFERENCES}

[1] ABDEL-SALAM, M. : Combined Method Based on Finite Differences and Charge Simulation for Calculating Electric Field, IEEE Transactions on Industry Applications 25 No. 6, (1989), 1060-1066.

[2] MALIK, N. H.: A review of the Charge Simulation Method and its Applications, IEEE Transactions on Electrical Insulation 24 No. 1 (1989), 3-20.

[3] VELiČKOVIĆ, D. M.-MILOVANOVIĆ, A.: Electrostatic Field of Cube Electrode, Serbian Journal of Electrical Engineering 1 No. 2 (2004), 187-198.

[4] VELICKOVIĆ, D. M : Equivalent Electrodes Method, Scientific Review, Belgrade No. 21-22 (1996), 207-248.

[5] DOBRICIC, M.: Distribution of Potential Surrounding Two Wire Lines with a Special Retrospect the Method of Complex Potential, PhD Dissertation, Technical faculty of Cacak, University of Kragujevac, 2008.

[6] MUSA, S. M.-SADIKU, M. N. : Analysis of Rectangular Coaxial Lines, IEEE Region 5 Technical Conference, April 20-21, Fayetteville, Arcansas, 2007.

[7] VELIČKOVIĆ, D. M.-MILOVANOVIĆ, A.: Approximate Calculation of Capacitance, Proceedings of VIII International IGTE Symposium on Numerical Field Calculation in Electrical Engineering, Graz, Austria, September 1998, pp. 339-344.

[8] LIN, W.-XIANG, Y.: Electrostatic Force on the Walls of a Rectangular Coaxial Line, Journal of Electrostatics 43 (1998), 275-283.
[9] RIBLET, H. J.: Expansion for the Capacitance of a Square in a Square with a Comparison, IEEE Trans. Microwave Theory Tech. 44 No. 2 (Feb 1996), 338-340.

[10] UITTEKER, E. T.-VATSON, D. N.: Kurs sovremennogo analiza (A Course of Modern Analysis), Fizmatgiz, Moskva, 1963. (in Russian)

[11] KAISER, K. L. : Transmission Lines, Matching, and Crosstalk, Taylor and Francis Group, Boca Raton, FL, 2006.

[12] Milovanović, A. M.-KOPRIVICA, B. M.-VESKOVIC, M. M.: The Capacitance of Two Wire Line with Rectangular Cross Section, International Conference on Electrical Systems Design and Technologies, Hammamet Tunisia, Nov 8-10, 2008, CD Proceedings.

[13] COSTAMAgNA, E.-FANNI, A.: Analysis of Rectangular Coaxial Structures by Numerical Inversion of the Schwarz-Christoffel Transformation, IEEE Trans. Magn. 28 (Mar 1992), 1454-1457.

[14] GREEN, H. E. : The Characteristic Impedance of Square Coaxial Line, IEE Transactions on Microwave Theory and Techniques MTT-11 (Nov 1963), 554-555.

[15] CHEN, T. S.: Determination of the Capacitance, Inductance, and Characteristic Impedance of Rectangular Lines, IRE Transactions on Microwave Theory and Techniques, 8 No. 5 (Sep 1960), 510-519.

[16] VELICKOVIĆ, D. M.-UHLMANN, F. H.-BRANDISKY, K.-STANTCHEVA, R. D.: Fundamentals of Modern Electromagnetics for Engineering, Technishe Universität Ilmenau, Germany, 2005.

[17] Software package Femlab, Version 2.3. 0.145, http://www.femlab.com/.

[18] Software package FEMM, http://femm.foster-miller.net/wiki/HomePage.

Received 29 December 2010

Alenka M. MILOVANOVIĆ was born in Cacak, Serbia on 11 December 1965. She received the MSc and PhD degrees in Electrical Engineering from the Faculty of Electronic Engineering of Nis in 1999 and from the Technical faculty of Cacak in 2007, respectively. Since 1991 she has been with the Department of Electronic and Electrical Engineering of Technical faculty of Cacak, where now work as Assistant Professor. Her research interest includes Computational electromagnetics and Applied electrostatics. She is the author/coauthor of more than 30 papers in journals and proceedings and the coauthor of three textbooks for students.

Miroslav M. BJEKIĆ was born on 28 August 1966 in Cacak, Serbia. He received MSc and PhD degrees in Electrical Engineering from the School of Electrical Engineering Belgrade (1995) and Technical Faculty of Cacak (2006), respectively. Since 1991 he has been working with the Department of Power Engineering of Technical Faculty of Cacak, where he works as Assistant Professor. His specialization: Computational electromagnetics, Micromachines and Step motors. He is the author/co-author of more than 50 papers in journals and proceedings, and the co-author of three textbooks for students. 